\title{
ACMG recommendations for standards for interpretation of sequence variations
}

\author{
ACMG Laboratory Practice Committee Working Group
}

These recommendations for the standardization of interpretation and reporting of sequence variations identified in the course of providing clinical laboratory services are intended (1) to provide a framework for the interpretation and reporting of such test results, and (2) to aid referring clinicians by educating them as to possible testing outcomes so that they may inform their patients and families appropriately.

\section{Interpretation of sequence variations}

Increasingly, clinical molecular laboratories are detecting novel sequence variations in the course of testing patient specimens. The certainty with which any given sequence variation is of clinical significance falls within a spectrum of interpretations, ranging from those in which the variation is almost certainly of clinical significance to those in which it is almost certainly not. The ACMG Laboratory Practice Committee Working Group on Standards for Interpretation of Sequence Variations recommends the following categories of sequence variations for the purposes of clinical reporting.

1. Sequence variation is previously reported and is a recognized cause of the disorder. Review of the literature, central mutation databases, e.g., Human Gene Mutation Database (HGMD), or the locus-specific database, to assess the current degree of certainty that the sequence variation is causative of the disorder should be undertaken prior to reporting. Concordance studies between phenotype and genotype within a family may provide acceptable criteria in the absence of more definitive functional studies.

2. Sequence variation is previously unreported and is of the type which is expected to cause the disorder. Examples include variation that is predicted to shift the mRNA reading frame; result in the introduction of a stop codon or a missense mutation of the normal stop codon; mutate the initiation codon (ATG); alter the sequence at a splice junction, particularly the invariant AG/GT nucleotides; or delete one or more exons in such a manner as to lead to a shift in the mRNA reading frame.

3. Sequence variation is previously unreported and is of the type which may or may not be causative of the disorder. Examples include a variation that is located within a splice consensus sequence, likely to produce a cryptic splice site, likely to affect transcription, or any missense mutation. A missense mutation that leads to nonconservative substitution of an evolutionarily conserved amino acid is more likely to be causative of the disorder than a missense mutation that leads to a conservative substitution or alters an amino acid that is not evolutionarily conserved. Family studies may clarify the pathologic or benign nature of missense mutations (see III, 1 below).

4. Sequence variation is previously unreported and is probably not causative of disease. An example includes sequence variation which does not produce an amino acid substitution and which is unlikely to produce a cryptic splice site.

5. Sequence variation is previously reported and is a recognized neutral variant. Review of the literature, central mutation databases, e.g., HGMD, and the locus-specific database, to assess the current degree of certainty that the sequence variation is a recognized neutral variant should be undertaken prior to reporting.

The extent to which a sequence variation in categories $2-4$ above is considered causative of disease may be influenced by a number of other parameters, including clinical presentation, the individual's risk of having the disorder, family history, other test results, and the sensitivity and specificity of the test being performed.

\section{Reporting of sequence variation}

The purpose of test reports is to state clearly the absence or presence of sequence variation and to set this information into clinical context in order to facilitate case management. Reports should be written in a style that is clear and informative to the clinician and does not require in-depth knowledge of molecular genetics to be interpretable and useful. Guidelines for report content are presented below.

1. Reports should clearly identify the gene analyzed, indicate the absence or presence of a sequence variation, and, if applicable, indicate the location of the sequence variation by nucleotide position, codons affected, and amino acid change(s). They should also include the fraction of exon sequence and intronexon boundary sequence analyzed.

2. Reports should clearly indicate the interpretative category of the test results (see above).

This guideline is designed primarily as an educational resource for medical geneticists and other health care providers to help them provide quality medical genetic services. Adherence to this guideline does not necessarily ensure a successful medical outcome. This guideline should not be considered inclusive of all proper procedures and tests or exclusive of other procedures and tests that are reasonably directed toward obtaining the same results. In determining the propriety of any specific procedure or test, the geneticist should apply his or her own professional judgment to the specific clinical circumstances presented by the individual patient or specimen. It may be prudent, however, to document in the patient's record the rationale for any significant deviation from this guideline. 
3. Reports should provide a discussion of the basis upon which the interpretation was made (e.g., sequence variation previously reported and a recognized cause of the disorder, or sequence variation previously unreported but based on additional clinical findings and/or testing of additional family members for the presence/absence of the sequence variation, is expected to cause the disorder).

4. Reports should indicate the methodology used in the analysis in sufficient detail to permit the clinician to determine how the test was performed, e.g., sequence variation screening by SSCP alone or in combination with targeted sequence analysis of all positives, sequence analysis of all genomic exons and promoters, sequence analysis of full length cDNA, percent of sequence analyzed, or other.

5. Reports should clearly indicate the limitations of the analysis performed, including the limitations of the methodology (e.g., "this SSCP analysis detects $85 \%$ of all SOD1 gene mutations") and the sensitivity of the test (e.g., "this test detects $85 \%$ of all mutations causing Norrie's disease"). This is particularly important in reports in which no sequence variation was identified, as the clinician must be provided with the information upon which to assess the individual's residual risk for the disorder. Reports should indicate whether the test sensitivity is based on published literature or studies carried out in the reporting laboratory. These reports may also include recommendations for additional studies.

6. Reports in which a sequence variation was identified should include information on penetrance, expressivity, etc., or state that no relevant data exist.

\section{Follow-up studies to ascertain the clinical significance of sequence variations}

It is recognized that clinical diagnostic laboratories may not have access to the resources necessary to definitively resolve genotype/phenotype correlations. However, follow-up activities may be useful to clarify this relationship and assist with risk assessment in specific cases, as presented below.

1. In cases in which the phenotype is de novo within the family, parental DNA can be evaluated for the presence or absence of the sequence variation identified previously in the proband. Assessment of the likely clinical significance of previously unreported sequence variations may be improved by the testing of a limited number of additional family members. Relationships, e.g., parentage and sibships, should be confirmed as appropriate.

Analysis of parental DNA is useful for clarifying the relationship between sequence variation and phenotype in all Mendelian disorders, no matter what the inheritance pattern. For X-linked recessive and autosomal dominant traits, absence of the sequence variation in either parent significantly increases the likelihood that the sequence variation is causative of the proband's phenotype. Conversely, the presence of the sequence variation in both an unaffected parent and the proband is of uncertain interpretation. Consideration of age of onset and phenotypic variation should be appropriately evalu- ated in this assessment. Entry of such sequence variation in locus-specific databases is encouraged.

2. Sequence variations, independent of clarification of their causation status, may be utilized in classical linkage analysis for carrier, predictive, and prenatal testing. In cases in which there is a family history of the disorder, parents, affected and unaffected siblings, and nuclear and extended family members can be evaluated for the presence of the sequence variation and the results subjected to linkage analysis. The accuracy of the analysis is subject to those sources of error inherent in linkage analyses, e.g., undetected recombination, locus heterogeneity, and availability of appropriate family members for testing.

3. In recessive disorders, inheritance of two sequence variants in trans should be confirmed by analysis of parental DNA. Association of two variants in cis removes the possibility that they are by themselves a complete explanation of an autosomal recessive phenotype. Also, apparent homozygosity for a sequence variant could be caused by compound heterozygosity for a sequence variant and a large deletion. This situation may be recognized by parental DNA analysis and, if necessary, confirmation of paternity.

When the causation status of a sequence variant is indeterminate, laboratories should consider carrying out familial concordance studies in an effort to clarify causation status. In addition, in certain instances laboratories might establish collaborative relationships with research facilities to determine genotype/phenotype correlations, such as through functional studies. Note that as of May 2000 functional studies have not been utilized by diagnostic laboratories.

\section{Clinical and technical validation of sequence variation detection}

Sequence analysis for clinical service may be provided only by laboratories holding current CLIA licensure. The ACMG recommends that interpretation and reporting of clinical molecular genetic data be limited to qualified providers such as those certified in the medical specialty of clinical molecular genetics by the American Board of Medical Genetics. Tests must be accepted as clinically useful (clinical validation) and must be technically validated by the service provider.

Approved by the ACMG Board of Directors by unanimous mail vote June 2000.

(C) AMERICAN COLLEGE OF MEDICAL GENETICS, 2000

9650 Rockville Pike, Bethesda, Maryland 20814-3998

Phone 301-530-7127, Fax 301-571-1895

ACMG Laboratory Practice Committee Working Group

Haig H. Kazazian, Jr., MD, Chair

University of Pennsylvania, Philadelphia

Corinne D. Boehm, MS

Johns Hopkins Hospital, Baltimore

William K. Seltzer, PhD

Athena Diagnostics, Inc., Worcester, MA

Helpful suggestions from the following are gratefully acknowledged: Arthur L. Beaudet, MD; Richard G.H. Cotton, DSc; Garry R. Cutting, MD; Jeffrey A. Kant, MD, PhD; Bradley W. Popovich, PhD; Thomas W. Prior, PhD; Carolyn Sue Richards, PhD. 